\title{
Compact stars with a small electric charge: the limiting radius to mass relation and the maximum mass for incompressible matter
}

\author{
José P. S. Lemos ${ }^{1, \mathrm{a}}$, Francisco J. Lopes ${ }^{1, \mathrm{~b}}$, Gonçalo Quinta ${ }^{1, \mathrm{c}}$, Vilson T. Zanchin ${ }^{2, \mathrm{~d}}$ \\ ${ }^{1}$ Departamento de Física, Centro Multidisciplinar de Astrofísica, CENTRA, Instituto Superior Técnico, IST, Universidade de Lisboa, UL, \\ Av. Rovisco Pais 1, 1049-001 Lisbon, Portugal \\ ${ }^{2}$ Centro de Ciências Naturais e Humanas, Universidade Federal do ABC, Rua Santa Adélia 166, Santo André, SP 09210-170, Brazil
}

Received: 9 December 2014 / Accepted: 31 December 2014 / Published online: 17 February 2015

(C) The Author(s) 2015. This article is published with open access at Springerlink.com

\begin{abstract}
One of the stiffest equations of state for matter in a compact star is constant energy density and this generates the interior Schwarzschild radius to mass relation and the Misner maximum mass for relativistic compact stars. If dark matter populates the interior of stars, and this matter is supersymmetric or of some other type, some of it possessing a tiny electric charge, there is the possibility that highly compact stars can trap a small but non-negligible electric charge. In this case the radius to mass relation for such compact stars should get modifications. We use an analytical scheme to investigate the limiting radius to mass relation and the maximum mass of relativistic stars made of an incompressible fluid with a small electric charge. The investigation is carried out by using the hydrostatic equilibrium equation, i.e., the Tolman-Oppenheimer-Volkoff (TOV) equation, together with the other equations of structure, with the further hypothesis that the charge distribution is proportional to the energy density. The approach relies on Volkoff and Misner's method to solve the TOV equation. For zero charge one gets the interior Schwarzschild limit, and supposing incompressible boson or fermion matter with constituents with masses of the order of the neutron mass one finds that the maximum mass is the Misner mass. For a small electric charge, our analytical approximating scheme, valid in first order in the star's electric charge, shows that the maximum mass increases relatively to the uncharged case, whereas the minimum possible radius decreases, an expected effect since the new field is repulsive, aiding the pressure to sustain the star against gravitational collapse.
\end{abstract}

\footnotetext{
a e-mail: joselemos@ist.utl.pt

be-mail: franciscojoaolopes@ist.utl.pt

c e-mail: goncalo.quinta@ist.utl.pt

de-mail: zanchin@ufabc.edu.br
}

\section{Introduction}

Compact stars and their properties have been a theme of great relevance on several grounds. Chandrasekhar's well-known work [1] on the maximum mass for white dwarfs advanced the way to the understanding of the nature and structure of compact stars. By using a cold equation of state in which the degeneracy electron pressure is the most relevant form of pressure for the support of a white dwarf against gravitational collapse, a radius-mass relation for these stars was deduced, from the non-relativistic electron regime in relatively large white dwarfs up to the relativistic electron regime in the most compact stars. He found that as the radius of the star approached zero the mass would go to a maximum value of $1.44 M_{\odot}$, where $M_{\odot}$ is the sun's mass. This is the Chandrasekhar limit. It uses Newtonian gravitation. Landau [2] through heuristic arguments found that the mass limit for white dwarfs could be written as $M \sim M_{\mathrm{pl}}^{3} / m_{n}^{2}$ where $m_{n}$ is the neutron or the proton mass and $M_{\mathrm{pl}}$ is the Planck mass, $M_{\mathrm{pl}}=\sqrt{\hbar c / G}$, with $\hbar$ being the Planck constant, $G$ the Newton constant of gravitation, and $c$ the velocity of light, or, setting units such that $G=1$ and $c=1$, which we do from now on, one has $M_{\mathrm{pl}}=\sqrt{\hbar}$. Putting in the numerical values for $M_{\mathrm{pl}}$ and $m_{n}$, the mass $M$ of the star is about the Chandrasekhar mass limit $M \sim 1 M_{\odot}$. Landau further deduced that the stars should have a radius of about $\lambda_{e} M_{\mathrm{pl}} / m_{n}$, where $\lambda_{e}$ is the electron's Compton wavelength, $\lambda_{e}=\hbar / m_{e}, m_{e}$ being the electron's mass, giving a radius of the order of $5000 \mathrm{~km}$. He also found that there was another regime in which the star is composed of neutrons, supported by the degeneracy pressure of these particles, and has a maximum mass of about $M \sim M_{\mathrm{pl}}^{3} / m_{n}^{2}$. These neutron stars are much more compact with a radius $\lambda_{n} M_{\mathrm{pl}} / m_{n}, \lambda_{n}$ being the neutron's Compton wavelength, giving about $10 \mathrm{~km}$. For objects with a radius tending to zero one should use general relativity, rather than Newtonian gravitation. In general 
relativity a compact star can be defined neatly as a star that has a geometrical mass $M$ (or, $G M / c^{2}$ if one restores $G$ and c) comparable to its radius $R$, i.e., $R / M \sim a$, with $a$ a number not much bigger than 1 , say of order of 10 or less. For an extended star like the Sun one has $R / M_{\odot} \sim 5 \times 10^{5}$, while for a white dwarf $R / M_{\odot} \sim 3 \times 10^{3}$, and for a neutron star $R / M_{\odot} \sim 6$, showing that the latter is really compact. On a general relativistic basis, Oppenheimer and Volkoff [3] worked out further the nature and structure of neutron stars. Setting up a stiff equation of state for stellar matter made of neutrons they found roughly the results of Landau [2], namely, the mass limit is about $1 M_{\odot}$ and $R / M_{\odot} \sim 6$. This limit is called the Landau-Oppenheimer-Volkoff limit. Improvements have been made on these limits. Using a cold equation of state valid in the full range of highly compressed matter, the Harrison-Wheeler equation of state, the full set of equilibria in an $R-M$ relation were found, in particular, the two maxima masses corresponding to the Chandrasekhar and Landau-Oppenheimer-Volkoff limits appear naturally [4]. See also [5] for further discussion of compact stars and [6] for issues of their stability. These mass limits, as seen in the context of general relativity, appear because at these stages the energy associated to the pressure is so strong that its gravitating effect overwhelms the self-support effect.

The properties of these compact stars get modified if either the constituent material is altered or an alternative theory of gravitation is used. The constituent material can be altered by introducing or adding new matter with different properties or even adding a new field to the star. It is also well known that the introduction of new matter fields can be mimicked by modifications of the gravitational field. One example, even in Newtonian gravitation, is that the effects of the dark matter can be mimicked by modifying the gravitational field, e.g., in the MOND theories [7-9]. The same type of choice holds true in tensor theories of gravitation, since one can pass the excess of the gravitational field present in the left-hand side of the Einstein equations to their right hand side giving an effective energy-momentum tensor in the form of a new field; e.g., see [10]. If this dark matter inhabits the core of stars it can imprint onto compact stars new properties $[11,12]$. On the other hand, the structure of stars like neutron stars in alternative theories of gravity has been analyzed in EinsteinDilaton-Gauss-Bonnet gravity [13], Eddington-like theories [14], with the conclusion that more massive stars than in general relativity can form, in a quadratic gravity theory $[15$, $16]$ and in $f(R)$ theories $[17,18]$. Stars and compact stars in braneworlds have also been studied in [19-22].

In contrast to the fermion stars mentioned above, there are stars made of bosons, the boson stars, that can have a wide range of mass limits, namely, $M \sim M_{\mathrm{pl}}^{2} / m_{b}, M \sim$ $M_{\mathrm{pl}}^{3} / m_{b}^{2}$, or $M \sim M_{\mathrm{pl}}^{4} / m_{b}^{3}$, where $m_{b}$ is the mass of the boson that makes up the star [23-25]; see [26,27] for reviews.
These stars could have been formed in the beginning of the universe from the primordial gravitational collapse of the boson particles and have been proposed as alternatives to the usual compact objects [27], such as neutron stars and black holes, and also as part of the dark matter [28]. These stars can, in principle, be detected [29-31] and a way to tell the difference between fermionic and bosonic dark matter has been proposed in [32].

Now, the first compact star ever displayed in its full structure was a general relativistic star with a very stiff equation of state, a star made of an incompressible perfect fluid, i.e., $\rho(r)=$ constant, and isotropic pressure [where $\rho(r)$ is the energy density at the radius $r$ ] [33]. This interior Schwarzschild star solution is spherically symmetric and has a vacuum exterior. An incompressible equation of state is interesting from various aspects, since one can extract clean results and it also provides compactness limits. Furthermore, this incompressible fluid applies to both fermion and boson particles, as long as the fluid has an ultra-stiff equation of state that can be approximated by an incompressible state. The speed of sound $(\mathrm{d} p / \mathrm{d} \rho)^{1 / 2}$ in such a fluid is infinite, which is not allowed relativistically. Notwithstanding, it is believed that the interiors of dense stars, such as neutron stars, are of uniform density, or nearly so, and thus this simple case is of practical interest. Schwarzschild found that there was a limit, when the central pressure $p_{c}$ goes to infinity and that the star's radius to the mass limit is $R / M=9 / 4=2.25$, indeed a very compact star [33]. Volkoff [34] and Misner [35] rederived the Schwarzschild interior limit of $R / M=9 / 4$ using the convenient Tolman-Oppenheimer-Volkoff (TOV) equation, a differential equation for the pressure profile as a function of the other quantities [3]; see also [36]. In addition, Misner [35] even found a maximum mass for a given density of the incompressible fluid, the Misner mass. The Schwarzschild limit yields, for the same mass, a radius that is well below the radius of a neutron star, and also yields the most possible compact boson star. An incompressible star whose radius is below this limit will in principle collapse into a singularity leaving a black hole to the exterior.

One can ask if the Schwarzschild limit can be modified, allowing for instance a lower $R / M$ relation. As mentioned above, one way is to have some kind of different matter or a new field in the star, or an alternative theory of gravitation. Rather than introducing an alternative theory of gravitation one can opt for a fluid with non-isotropic pressure [37], for instance. Here we select to study the case in which we add a matter field to the existent matter. We consider matter with a small electric charge, introducing thus an additional electric field in addition to the usual matter and gravitational fields. This addition of an electric charge and an electric field to the Schwarzschild incompressible matter configurations brings insight to the configurations' overall struc- 
ture in more complex situations and its study in stars mimics other fields and possible alterations in the gravitational field.

Spheres with electric charge tend to be unstable. Since like charges repel each other, if in one way or another a charged cloud forms it will blow out at once due to the electrical repulsion. However, gravitation is universal and attractive. For a sphere with a small mass the electric repulsion is stronger than the gravitational pull and the matter suffers an expansion. On the other hand, for a highly massive sphere with a small quantity of charge the gravitational pull on the charged matter can overcome the electric repulsion and thus the system collapses. In between the two situations, one can have an equilibrium situation when the gravitational pull and the electric repulsion balance each other and the sphere stays static. In general, there is also matter pressure which adds to the balance of forces. For an extended star the pressure acts to counterbalance the gravitational field, but for highly compact stars the pressure acts as an energy source that adds to the star's mass and energy increasing in an overwhelming fashion the gravitational pull against its own pressure push. Thus, in the balance between the gravitational, electric and pressure fields, in highly compact stars electric charge can remain trapped within the stars. In case the compact stars supports some electric charge, their structure and properties are modified, and in particular, the radius-mass relations for the corresponding stars should change.

One important quantity that gives a measure of how much electric charge a star can support is the ratio of the mass $m$ to the charge $q$ of the main fundamental constituents of the star. For normal matter the net electric matter in a star is utterly negligible as the ratio of the proton mass $m_{p}$ to the proton charge $e$ is $m_{p} / e=10^{-18}$, giving thus $Q / M \simeq\left(m_{p} / e\right)^{2} \simeq 10^{-36}$, where $Q$ is the star's total charge [5] (see also [38]). However, stars can contain some dark matter in their interior, and of the several dark matter fluid candidates some could be electrically charged. Indeed, natural candidates to compose the dark matter are supersymmetric particles. The lightest supersymmetric particles that make the bulk of dark matter should be neutral, one possible candidate is the neutralino [39], however, some of these particles could be electrically charged. The mass $m$ to charge $q$ ratio of these supersymmetric particles are much higher than the baryonic mass to charge ratio, indeed current supergravity theories indicate that some particles can have a ratio of one. For a $m / q \sim 0.1-0.3$ one has $Q / M \simeq 0.01-0.1$, a small but non-negligible electric charge. Thus, if dark matter populates the interior of stars, and some of it is made of electrically charged particles there is the possibility that stars have some electric charge.

That electric charge can influence the structure of a compact star was proposed earlier by Bekenstein [40] who wrote the appropriate TOV equation. Some electric compact con- figurations with an incompressible equation of state for the matter were studied numerically and the corresponding generalized Schwarzschild limit, i.e., central pressure going to infinity in these configurations, was analyzed [4144]. Other equations of state for electrically charged matter, such as polytropic equations, were used in [45-47], where star configurations and their structure were studied and the Schwarzschild electric limit for the given equation of state and for a given charge was considered. In particular, in [45] it was argued that upon gravitational collapse a star could retain, significantly, part of its electric charge. This electric charge excess could remain trapped in the final configuration, be it a highly compact star or a black hole. Other interesting equations of state were proposed and studied in $[48,49]$, and in [50] where electrically charged dust was studied. Electrically charged boson stars have also been studied and their properties analyzed $[51,52]$. Bounds on the radius to mass relation for uncharged stars have been put forward in [53]; see also [54-57]. Bounds on the radius to mass relation for charged stars have been set in [58]; see also [59-61]. It is also worth mentioning some work on charged Newtonian stars. That compact stars could exist was even noticed in the 18th century. A subset of these Newtonian compact stars are the dark stars of Mitchell, also mentioned later by Laplace; see [62]. The Chandrasekhar white dwarfs of very small radius, including the one with zero radius that gives the Chandrasekhar maximum mass, are also Newtonian compact stars. Indeed the stars that have very small radii, from the gravitational radius to zero radius, provide instances of the dark stars of Mitchell and Laplace. Of course, these stars cannot exist in nature as for strong gravitational fields Newtonian gravitation is invalid. That compact Newtonian stars could be electrified was raised in [63] and further developed in [64]. Turning the table around, the real analog of dark stars are the quasiblack holes considered in, e.g., [49].

In order to understand the effects of a small electric charge on a star, and in particular, on the interior Schwarzschild limit, we use an analytical scheme and investigate the limiting radius to mass relation and the maximum mass of relativistic compact stars made of an electrically charged incompressible fluid. The investigation is carried out using the hydrostatic equilibrium equation, i.e., the TOV equation, and the other structure equations, with the further hypothesis that the charge distribution is proportional to the energy density. The approach relies on Volkoff and Misner's method [34,35] to solve the TOV equation. For zero charge one gets the interior Schwarzschild limit and under certain assumptions one gets the Misner mass. Our analysis for stars with a small electric charge shows that the maximum mass increases relatively to the uncharged case, whereas the minimum possible radius decreases, an expected effect since the new field is repulsive aiding the pressure to sustain the star against gravitational collapse. 
The paper is organized as follows. In Sect. 2 we give the general relativistic equations, the equations of structure for a static spherically symmetric configuration, the equations of state for energy density and charge density, and discuss the exterior spacetime and the boundary conditions. In Sect. 3 we review the pure, uncharged, interior Schwarzschild limit using the Volkoff and Misner's formalism to set the nomenclature. We also give the Misner mass. In Sect. 4 we study analytically the interior electric Schwarzschild limit using the Volkoff and Misner formalism and give the electric correction to the Misner mass. In Sect. 5 we conclude. In the appendix we derive some equations necessary in our study.

\section{The full set of equations and boundary conditions}

\subsection{Basic general relativistic equations}

We are interested in analyzing highly compacted charged spheres as described by the Einstein-Maxwell equations with charged matter. We set $G=1$ and $c=1$. The field equations are

$G_{\mu \nu}=8 \pi T_{\mu \nu}$

$\nabla_{\nu} F^{\mu \nu}=4 \pi J^{\mu}$

where Greek indices are spacetime indices running from 0 to 3 , with 0 being a time index. The Einstein tensor $G_{\mu \nu}$ is defined as $G_{\mu \nu}=R_{\mu \nu}-\frac{1}{2} g_{\mu \nu} R$, where $R_{\mu \nu}$ is the Ricci tensor $R_{\mu \nu}, g_{\mu \nu}$ is the metric tensor, and $R$ the Ricci scalar. The Faraday-Maxwell tensor $F_{\mu \nu}$ is defined in terms of an electromagnetic four-potential $A_{\mu}$ by $F_{\mu \nu}=\partial_{\mu} A_{\nu}-\partial_{\nu} A_{\mu}$. Equation (1) is the Einstein equation, stating the relation between the Einstein tensor and the energy-momentum tensor $T_{\mu \nu}$. Here $T_{\mu \nu}$ is written as a sum of two terms,

$T_{\mu \nu}=E_{\mu \nu}+M_{\mu \nu}$

$E_{\mu \nu}$ is the electromagnetic energy-momentum tensor, which is given in terms of the Faraday-Maxwell tensor $F_{\mu \nu}$ by the relation

$E_{\mu \nu}=\frac{1}{4 \pi}\left(F_{\mu}^{\gamma} F_{\nu \gamma}-\frac{1}{4} g_{\mu \nu} F_{\gamma \beta} F^{\gamma \beta}\right)$.

$M_{\mu \nu}$ represents the matter energy-momentum tensor and we assume it is the energy-momentum tensor of a perfect fluid, namely,

$M_{\mu \nu}=(\rho+p) U_{\mu} U_{v}+p g_{\mu \nu}$,

with $\rho$ and $p$ being the energy density and the pressure of the fluid, respectively, and $U_{\mu}$ being the fluid four-velocity.
Equation (2) is the Maxwell equation, stating the proportionality between the covariant derivative $\nabla_{v}$ of the FaradayMaxwell tensor $F_{\mu \nu}$ and the electromagnetic four-current $J_{\mu}$. For a charged fluid, this current is given in terms of the electric charge density $\rho_{e}$ by

$J^{\mu}=\rho_{e} U^{\mu}$

The other Maxwell equation $\nabla_{[\alpha} F_{\beta \gamma]}=0$, where $[\ldots]$ means antisymmetrization, is automatically satisfied.

\subsection{Equations of structure}

The line element for a static spherically symmetric spacetime is of the form

$\mathrm{d} s^{2}=-B(r) \mathrm{d} t^{2}+A(r) \mathrm{d} r^{2}+r^{2}\left(\mathrm{~d} \theta^{2}+\sin ^{2} \theta \mathrm{d} \phi^{2}\right)$,

where $t, r, \theta$, and $\phi$ are the usual Schwarzschild-like coordinates, and the metric potentials $A(r)$ and $B(r)$ are functions of the radial coordinate $r$ only. The assumed spherical symmetry of the spacetime implies that the only nonzero components of a purely electrical Faraday-Maxwell tensor $F^{\mu \nu}$ are $F^{t r}$ and $F^{r t}$ with $F^{t r}=-F^{r t}$ and where $F^{t r}$ is a function of the radial coordinate $r$ alone, $F^{t r}=F^{t r}(r)$. The other components of $F^{\mu v}$ are identically zero. It is advantageous to define the total electric charge $q(r)$ inside a spherical surface labeled by the radial coordinate whose value is $r$ by

$q(r)=F^{t r} r^{2} \sqrt{A(r) B(r)}$.

That is, one swaps $F^{t r}$ for $q(r)$. It is also opportune to define a new quantity $m(r)$ in such a way that

$\frac{1}{A(r)}=1-\frac{2 m(r)}{r}+\frac{q^{2}(r)}{r^{2}}$.

That is, one swaps $A(r)$ for $m(r)$. The new function $m(r)$ represents the gravitational mass inside the sphere of radial coordinate $r$.

One of the Einstein equations can be substituted by the contracted Bianchi identity $\nabla_{\mu} T^{\mu \nu}=0$, which gives

$\frac{\mathrm{d} B(r)}{\mathrm{d} r}=\frac{B(r)}{p(r)+\rho(r)}\left[\frac{q(r)}{2 \pi r^{4}} \frac{\mathrm{d} q(r)}{\mathrm{d} r}-2 \frac{\mathrm{d} p(r)}{\mathrm{d} r}\right]$,

a differential equation for $B, q$, and $p$. The Einstein equations also give a differential equation for $B(r)$ alone, i.e.,

$$
\begin{gathered}
\left(1-\frac{2 m(r)}{r}+\frac{q^{2}(r)}{r^{2}}\right)\left[1+\frac{r}{B(r)} \frac{\mathrm{d} B(r)}{\mathrm{d} r}\right] \\
=1+8 \pi r^{2}\left[p(r)-\frac{q^{2}(r)}{8 \pi r^{4}}\right] .
\end{gathered}
$$


Now, we are ready to write the other three equations in a form we want to use. One finds that another of Einstein equations gives a differential equation for $m(r)$, i.e.,

$$
\frac{\mathrm{d} m(r)}{\mathrm{d} r}=4 \pi \rho(r) r^{2}+\frac{q(r)}{r} \frac{\mathrm{d} q(r)}{\mathrm{d} r} .
$$

Since $m(r)$ represents the gravitational mass inside the sphere of radial coordinate $r$, then Eq. (12) represents the energy conservation as measured in the star's frame. The only nonvanishing component of the Maxwell Eq. (2) is given by

$$
\frac{\mathrm{d} q(r)}{\mathrm{d} r}=4 \pi \rho_{e}(r) r^{2} \sqrt{1-\frac{2 m(r)}{r}+\frac{q^{2}(r)}{r^{2}}} .
$$

Finally, replacing Eq. (13) and the conservation equation (10) into Eq. (11) yields

$$
\begin{aligned}
\frac{\mathrm{d} p}{\mathrm{~d} r}= & -(p+\rho) \frac{\left(4 \pi p r+m / r^{2}-q^{2} / r^{3}\right)}{\left(1-2 m / r+q^{2} / r^{2}\right)} \\
& +\rho_{e} \frac{q / r^{2}}{\sqrt{1-2 m / r+q^{2} / r^{2}}},
\end{aligned}
$$

where to simplify the notation we have dropped the functional dependence, i.e., $m(r)=m, q(r)=q, \rho(r)=\rho, p(r)=p$, and $\rho_{e}(r)=\rho_{e}$. Equation (14) is the TOV equation modified by the inclusion of electric charge [40] (see also [47]). The system of Eqs. (11)-(14) is the system we were looking for. We need now to specify the equation of state and the equation for the charge density profile.

\subsection{Equation of state and the charge density profile}

In the present model there are six unknown functions: $B(r)$, $m(r), q(r), \rho(r), p(r)$, and $\rho_{e}(r)$; and just four equations: Eqs. (11)-(14). Additional relations are obtained from a model for the cold fluid, which furnishes relations among the pressure and the energy density. For an electrically charged fluid, a relation defining the electric charge distribution is also needed.

Here we assume an incompressible fluid, i.e.,

$\rho(r)=$ constant.

So the energy density is constant along the whole star.

Following [45] (see also [47]), we assume a charge density proportional to the energy density,

$\rho_{e}=\alpha \rho$,

where, in geometric units, $\alpha$ is a dimensionless constant which we call the charge fraction. The charge density along the whole star is thus constant as well. Other equations for the charge distribution could be considered, as more charge concentration on the core, or more charge on the outer layers; see, e.g., [41-43]. An equation for the charge density as the one given in (16) should be commented on. In principle, the permittivity $\varepsilon$ of such a medium cannot be equal to the vacuum permittivity $\varepsilon_{\mathrm{vac}}=1$. Such a $\varepsilon \neq \varepsilon_{\mathrm{vac}}$ certainly has influence on the electrostatic Eq. (13) and also possibly on the stress-energy tensor $E_{\mu \nu}$ of the electromagnetic field given in Eq. (4). Here we consider that the medium is such that its response to an applied electric field is low enough so that one can consider that it has a permittivity equal to the vacuum permitivity. In a more detailed account permittivity effects should be considered.

We have now four equations: Eqs. (11)-(14); and four unknowns: $B(r), m(r), q(r)$, and $p(r)$, as $\rho$ and $\rho_{e}$ are given in (15) and (16), respectively. The resulting set of equations constitute the complete set of structure equations which, with some appropriate boundary conditions, can be solved simultaneously. We are not going to solve it (see [44]). Here we use this system of equations to find the Schwarzschild interior limit for the small charge case.

\subsection{The exterior vacuum region to the star and the boundary conditions}

The conditions at the center of the star are that $m(r=0)=0$, $q(r=0)=0$, and $A(r=0)=1$ to avoid any type of singularities, and that $p(r=0)=p_{c}, \rho(r=0)=\rho_{c}$, and $\rho_{e}(r=0)=\rho_{e c}$, where $p_{c}$ is the central pressure, $\rho_{c}$ is the central energy density, and $\rho_{e c}$ is the central charge distribution, the latter two having the same constant values throughout the star [see Eqs. (15) and (16)].

The interior solution is matched at the surface to the exterior Reissner-Nordström spacetime, with metric given by

$\mathrm{d} s^{2}=-F(r) \mathrm{d} T^{2}+\frac{\mathrm{d} r^{2}}{F(r)}+r^{2}\left(\mathrm{~d} \theta^{2}+\sin ^{2} \theta \mathrm{d} \phi^{2}\right)$,

where

$F(r)=1-\frac{2 M}{r}+\frac{Q^{2}}{r^{2}}$,

with the outer time $T$ being proportional to the inner time $t$, and $M$ and $Q$ being the total mass and the total charge of the star, respectively.

At the surface of the star one has a vanishing pressure, i.e., $p(r=R)=0$. The boundary conditions at the surface of the star are then $B(R)=1 / A(R)=F(R), m(R)=M$, $q(R)=Q$, besides $p(R)=0$.

An important quantity for the exterior metric is the gravitational or horizon radius $r_{+}$of the configuration. The Reissner-Nordström metric, given through Eqs. (17) and (18), has 
$r_{+}=M+\sqrt{M^{2}-Q^{2}}$.

as the solution for its own gravitational radius.

\section{The interior Schwarzschild limit: the zero charge case}

\subsection{Equations}

Before we treat the small charge case analytically, we consider the exact Schwarzschild interior solution as given by Volkoff [34] and displayed later in Misner's lectures [35]. For this we put $q=0$ in Eqs. (11)-(14). Equation (11) is of no direct interest here; Eq. (12) gives

$\frac{\mathrm{d} m(r)}{\mathrm{d} r}=4 \pi \rho(r) r^{2}$,

Equation (13) is trivially satisfied in this case, and finally, Eq. (14) simplifies to

$\frac{\mathrm{d} p}{\mathrm{~d} r}=-(p+\rho) \frac{4 \pi p r+m / r^{2}}{1-2 m / r}$.

Since, by Eq. (15), the density is constant we can integrate Eq. (20), obtaining

$m(r)=\frac{4}{3} \pi \rho r^{3}, \quad 0 \leq r \leq R$,

where $R$ is the star radius and we have imposed that there is no point mass in the center. Defining a characteristic length $R_{c}$ by

$R_{c}^{2}=\frac{3}{8 \pi \rho}$,

we can rewrite the mass function, Eq. (22), as

$m(r)=\frac{1}{2} \frac{r^{3}}{R_{c}^{2}}, \quad 0 \leq r \leq R$.

Interchanging $\rho$ and $R_{c}$ as necessary and noting that $2 \rho R_{c}^{2}=$ $\frac{3}{4 \pi}$ we get from Eq. (21),

$\frac{\mathrm{d} p}{\mathrm{~d} r}=-\frac{(p+\rho)(3 p+\rho)}{\rho} \frac{1}{2 R_{c}^{2}} \frac{r}{1-r^{2} / R_{c}^{2}}$.

3.2 The interior Schwarzschild limit: the $R$ and $M$ relation and the minimum radius

Equation (25) is separable and can be integrated as

$\int \mathrm{d} p \frac{\rho}{(\rho+p)(\rho+3 p)}=-\frac{1}{2} \int \mathrm{d} r \frac{r}{R_{c}^{2}} \frac{1}{1-r^{2} / R_{c}^{2}}$, with the boundary condition that the surface of the star $R$ has zero pressure, i.e.,

$p(R)=0$.

Defining a new radial coordinate $\chi$ by

$r=R_{c} \sin \chi$,

Equation (26) can be put in the form

$\int d p \frac{\rho}{(\rho+p)(\rho+3 p)}=-\frac{1}{2} \int d(\ln \cos \chi)$,

subjected to the boundary condition

$p\left(\chi_{s}\right)=0$,

where $\chi_{s}$ is given through

$R=R_{c} \sin \chi_{s}$

Integrating Eq. (29), subjected to the boundary condition (30), yields the pressure

$p=\rho \frac{\cos \chi-\cos \chi_{s}}{3 \cos \chi_{s}-\cos \chi}$.

The central pressure, $p_{c}$ is the pressure computed at zero radius $r=0$, i.e., $\chi=0$, so that

$p_{c}=\rho \frac{1-\cos \chi_{s}}{3 \cos \chi_{s}-1}$.

This blows up,

$p_{c} \rightarrow \infty$ when $\cos \chi_{s} \rightarrow \frac{1}{3}$.

This is equivalent to

$\sin ^{2} \chi_{s}=\frac{8}{9}$.

Now, Eqs. (24) and (28) allow us to write

$M=\frac{1}{2} R \sin ^{2} \chi_{s}$,

where $M \equiv m(R)$ is the star's total mass. Thus, Eqs. (35) and (36) yield

$\frac{R}{M}=\frac{9}{4}$

Equation (37) is the Schwarzschild limit found in [33]. 


\subsection{Misner mass bound}

Following Misner [35] we can also display a mass bound. Equation (24) gives

$M=\frac{1}{2} \frac{R^{3}}{R_{c}^{2}}$.

Eliminating $R$ in Eqs. (37) and (38), and noting that $p_{c} \leq \infty$, one gets the mass bound

$M \leq \frac{1}{2}\left(\frac{8}{9}\right)^{3 / 2} R_{c}$

To get a mass we have to have a density and thus an $R_{c}$. One can make sense of a constant density if one takes it as the density at which matter is almost incompressible and the pressure throughout the star is very high. If the fluid is an ideal gas this happens when the particles have relativistic velocities of the order 1. For fermions this happens when the Fermi levels are near the rest mass $m_{n}$ of the fermions, neutrons say, while for bosons this means that the gas temperatures are of the order of the rest mass $m_{b}$ of the particles. This gives, for both fermions and bosons, a density of one particle per cubic Compton wavelength. That is, for a particle with mass $m$ and Compton wavelength $\lambda$ given by $\lambda=\hbar / m$ the density is $\rho \sim m^{4} / \hbar^{3}$. In the case of a star composed of neutrons, Misner [35] obtains

$M \leq 1.5 M_{\odot}$,

where $M_{\odot}$ is the Sun's mass. This bound is similar to the Chandrasekhar limit $M_{\text {Chandrasekhar }}=1.44 M_{\odot}$, or the Oppenheimer-Volkoff mass, $M_{\mathrm{OV}} \simeq 1 M_{\odot}$, both found for equations of state different from the one used here and through totally different means.

\section{The electrically charged interior Schwarzschild limit: the small charge case}

4.1 Equations: perturbing with a small electric charge

\subsubsection{Expansion in the electric charge parameter $\alpha$}

We are going to solve Eqs. (12), (13), and (14), treating the charge $q(r)$ as a small perturbation, thus assuming $\alpha$ small. To do so, we note that the solutions for the mass and the charge will be of the form

$$
\begin{aligned}
q(r) & =q_{1}(r), \\
m(r) & =m_{0}(r)+m_{1}(r),
\end{aligned}
$$

where we are assuming that the non-perturbed charge is zero $q_{0}(r)=0, m_{0}(r)$ is the mass of the uncharged star given by Eq. (20), or Eq. (22), and $q_{1}(r)$ and $m_{1}(r)$ are the perturbed small charge and mass functions to be determined. The pressure is also assumed to be given by the expansion

$p(r)=p_{0}(r)+p_{1}(r)$,

where $p_{0}$ is the pressure in the uncharged case, given by Eq. (25), or (32), and $p_{1}(r)$ is the perturbation induced in the pressure when a small charge is considered. Note that, while the boundary condition for the non-charged star was simply $p(R)=p_{0}(R)=0$, the boundary condition for the charged star becomes

$p_{0}(R)+p_{1}(R)=0$.

At this point, it will prove useful to introduce the dimensionless variable

$x=\frac{r}{R_{c}}$,

where $R_{c}$ is the characteristic length defined in Eq. (23). The expressions for the mass, charge, and pressure in this new variable are generically defined as

$m(x)=\frac{m(r)}{R_{c}}, \quad q(x)=\frac{q(r)}{R_{c}}, \quad p(x)=\frac{p(r)}{\rho}$.

From Eq. (45) we defined $x_{s}$ as the $x$ at the surface, so that

$x_{s}=\frac{R}{R_{c}}$.

Accordingly, we define

$m\left(x_{s}\right)=\frac{M}{R_{c}}, \quad q\left(x_{s}\right)=\frac{Q}{R_{c}}, \quad p\left(x_{s}\right)=\frac{p(R)}{\rho}$.

\subsubsection{Calculation of the perturbed charge distribution $q_{1}$}

Expanding Eq. (13) for small $\alpha$, we get in the $x$ variable that

$\frac{\mathrm{d} q_{1}}{\mathrm{~d} x}=\frac{3}{2} \alpha \frac{x^{2}}{\sqrt{1-x^{2}}}$,

up to first order in $\alpha$. Solving the above equation subject to the condition $q_{1}(0)=0$ and expressing the solution in terms of the variable $x$, results in

$q_{1}(x)=\frac{3}{4} \alpha\left(\arcsin x-x \sqrt{1-x^{2}}\right)$. 


\subsubsection{Calculation of non-perturbed and perturbed masses}

The unperturbed mass $m_{0}$ can now be expressed simply as

$m_{0}(x)=\frac{x^{3}}{2}$

One can also find an expression for $m_{1}$, namely,

$m_{1}(x)=\frac{3}{8} \alpha^{2}\left(3 x-x^{3}-3 \sqrt{1-x^{2}} \arcsin x\right)$.

Indeed, from Eqs. (12) and (42), it is clear that the equation for the perturbed mass $m_{1}$ is given by

$\frac{\mathrm{d} m_{1}}{\mathrm{~d} x}=\frac{q_{1}}{x} \frac{\mathrm{d} q_{1}}{\mathrm{~d} x}$.

Inserting Eq. (50) into Eq. (53), we can integrate it using the boundary condition that the total mass at the center of the star is $m(0)=0$, which implies that $m_{1}(0)=0$ since Eq. (22), or Eq. (51), satisfies $m_{0}(0)=0$. Doing this, we are led to Eq. (52).

\subsubsection{Equations for the pressures, solution for the zeroth order pressure, and calculation of the perturbed pressure at the star's radius}

(i) Equations for the pressures

To find the equations for the pressures $p_{0}(x)$ and $p_{1}(x)$, we begin by expressing Eq. (14) for the total pressure in terms of the variable $x$ given in Eq. (45),

$$
\begin{aligned}
\frac{\mathrm{d} p}{\mathrm{~d} x}= & -\frac{(1+p(x))\left(3 p(x) x / 2+m(x) / x^{2}-q^{2}(x) / x^{3}\right)}{1-2 m(x) / x+q^{2}(x) / x^{2}} \\
& +\frac{\alpha q}{x^{2} \sqrt{1-2 m(x) / x+q^{2}(x) / x^{2}}}
\end{aligned}
$$

Now we can expanded the right side of the above equation in powers of $\alpha$ and retain the two lowest terms. By doing so, and using the expansion (43) on the left side of Eq. (54) and Eqs. (41) and (42) on the right hand side, we can equate the terms in equal powers of $\alpha$, thus obtaining two differential equations. The first one, obtained from the 0 th power in $\alpha$ is

$$
\frac{\mathrm{d} p_{0}(x)}{\mathrm{d} x}=-\frac{\left(1+p_{0}(x)\right)\left(3 p_{0}(x) x / 2+m_{0}(x) / x^{2}\right)}{1-2 m_{0}(x) / x}
$$

which is simply the differential equation for the unperturbed pressure. The second differential equation, to first order in $\alpha^{2}$, is

$$
\begin{aligned}
\frac{\mathrm{d} p_{1}}{\mathrm{~d} x}= & \frac{\alpha q_{1}}{x^{2} \sqrt{1-2 m_{0} / x}}-\frac{\left(1+p_{0}\right)\left(3 p_{0} x / 2+x / 2\right) f_{1}}{\left(1-2 m_{0} / x\right)^{2}} \\
& -\frac{p_{1}\left(3 p_{0} x / 2+x / 2\right)+\left(1+p_{0}\right)\left(3 p_{1} x / 2+f_{2}\right)}{\left(1-2 m_{0} / x\right)},
\end{aligned}
$$

which is the differential equation for the perturbed pressure $p_{1}$, where, again, to shorten equations we have dropped the dependence of variables $p_{1}, p_{0}, m_{1}, m_{0}$, and $q_{1}$ on $x$, and we have also defined the auxiliary functions $f_{1}=f_{1}(x)$ and $f_{2}=f_{2}(x)$ by

$f_{1}(x)=\frac{2 m_{1}(x)}{x}-\frac{q_{1}^{2}(x)}{x^{2}}$

and

$f_{2}(x)=\frac{m_{1}(x)}{x^{2}}-\frac{q_{1}^{2}(x)}{x^{3}}$.

Ultimately, we want to obtain an equation for the radius $R$ for which the central pressure blows up. From Eq. (43), the central pressure is $p(0)=p_{0}(0)+p_{1}(0)$. In the appendix we show that $p_{1}(0)$ is always finite. So we have to find a solution for the radius $R$ at which $p_{0}(0)$ blows up.

(ii) Solution for the zeroth order pressure

We start by obtaining a solution for $p_{0}$. Since the boundary condition has changed relatively to the uncharged case, it is now given by Eq. (44), we cannot use a priori the form (32) for $p_{0}$. We use the solution to Eq. (55) without specifying any boundary condition. In the variable $x$, this means

$p_{0}(x)=\frac{\sqrt{1-x^{2}}-A}{3 A-\sqrt{1-x^{2}}}$,

where $A$ is an integration constant. To see what this constant is, we insert the above equation into Eq. (44) and solve the resulting equation with respect to $A$. After expanding for the small charge parameter $\alpha$, and so for small $p_{1}$, we are led to

$A=\sqrt{1-x_{s}^{2}}\left(1+2 p_{1}\left(x_{s}\right)\right)$,

up to first order in the perturbed quantities. Then the expression for $p_{0}$, analogous to Eq. (32), becomes

$p_{0}(x)=\rho \frac{\sqrt{1-x^{2}}-\sqrt{1-x_{s}^{2}}\left(1+2 p_{1}\left(x_{s}\right)\right)}{3 \sqrt{1-x_{s}^{2}}\left(1+2 p_{1}\left(x_{s}\right)\right)-\sqrt{1-x^{2}}}$.

Since $p_{0}(x)$ depends on $p_{1}\left(x_{s}\right)$ in the denominator, we have to find $p_{1}\left(x_{s}\right)$, i.e., we have to calculate the perturbed pressure at the star's radius. 
(iii) Calculation of the perturbed pressure at the star's radius The equation for $p_{1}$, Eq. (56), cannot be solved analytically for all $x$. However, we are only interested in the value of $p_{1}$ at the surface of the star. At this particular radius it is possible to obtain the exact value of the perturbed pressure without ever solving Eq. (56). The reason for this is the fact that at the star's radius the pressure $p\left(x_{s}\right)=p_{0}\left(x_{s}\right)+p_{1}\left(x_{s}\right)$ is zero. Therefore, we can insert the boundary condition $p\left(x_{s}\right)=0$ in the exact derivative of the pressure given by Eq. (54) and expand the resulting equation for small $\alpha$, giving

$$
\begin{aligned}
\left.\frac{\mathrm{d} p}{\mathrm{~d} x}\right|_{x=x_{s}}= & -\frac{m_{0}\left(x_{s}\right)}{x_{s}^{2}\left(1-2 m_{0}\left(x_{s}\right) / x_{s}\right)} \\
& -\frac{m_{0}\left(x_{s}\right)}{1-2 m_{0}\left(x_{s}\right) / x_{s}}\left(\frac{f_{1}\left(x_{s}\right)}{x_{s}^{2}}+\frac{f_{2}\left(x_{s}\right)}{m_{0}\left(x_{s}\right)}\right),
\end{aligned}
$$

up to first order, and where the auxiliary functions $f_{1}(x)$ and $f_{2}(x)$ are given by Eqs. (57) and (58), respectively. Using the expansion (43) on the left side of Eq. (62), one can clearly see that there is a compatibility condition which must be physically required, namely that, at the star surface, the first term on the right side of Eq. (62) must be equal to Eq. (55) and the second term equal to Eq. (56). Hence, we arrive at the two equations

$$
\frac{3}{2} p_{0}^{2}\left(x_{s}\right)+2 p_{0}\left(x_{s}\right)=0
$$

and

$\frac{3}{2} p_{1}\left(x_{s}\right) p_{0}\left(x_{s}\right) x_{s}+p_{0}\left(x_{s}\right) f_{2}\left(x_{s}\right)=0$.

These equations give two different solutions, namely, $p_{0}\left(x_{s}\right)=0$ and $p_{1}\left(x_{s}\right)=0$, or $p_{0}\left(x_{s}\right)=-\frac{4}{3}$ and $p_{1}\left(x_{s}\right)=-\frac{2}{3} x_{s}^{-1} f_{2}\left(x_{s}\right)$. This latter solution does not satisfy the boundary condition (44) so the unique valid solution is given by

$p_{0}\left(x_{s}\right)=0$,

and

$p_{1}\left(x_{s}\right)=0$.

\subsubsection{Equation for the minimum radius}

We see that the central pressure $p_{0}(0)$ given in Eq. (61) is divergent when the following condition holds:

$3 \sqrt{1-x_{s}^{2}}\left(1+2 p_{1}\left(x_{s}\right)\right)=1$.
Expanding it in $\alpha^{2}$ we arrive at

$\frac{x_{s}}{m_{0}\left(x_{s}\right)}=\frac{9}{4}-\frac{9}{8} p_{1}\left(x_{s}\right)$

valid in first order in $\alpha^{2}$. Using the expansion provided by Eq. (42), it can be shown that to first order in $\alpha^{2}$, we have

$\frac{x_{s}}{m\left(x_{s}\right)}=\frac{x_{s}}{m_{0}\left(x_{s}\right)}-x_{s} \frac{m_{1}\left(x_{s}\right)}{m_{0}^{2}\left(x_{s}\right)}$.

Upon substituting Eq. (69) into Eq. (68) we conclude that

$\frac{x_{s}}{m\left(x_{s}\right)}=\frac{9}{4}-\left(\frac{9}{8} p_{1}\left(x_{s}\right)+x_{s} \frac{m_{1}\left(x_{s}\right)}{m_{0}^{2}\left(x_{s}\right)}\right)$.

Now, the minimum star radius $R$ will not be just $\sqrt{8 / 9} R_{c}$ but will have corrections of order $\alpha^{2}$. These corrections will induce changes of the order $\alpha^{4}$ in Eq. (70). Thus, we can set $x_{s}=\sqrt{8 / 9}$ in Eq. (70), i.e.,

$\frac{x_{s}}{m\left(x_{s}\right)}=\frac{9}{4}-\left(\frac{9}{8} p_{1}\left(x_{s}\right)+\sqrt{\frac{8}{9}} \frac{m_{1}\left(x_{s}\right)}{m_{0}^{2}\left(x_{s}\right)}\right)$,

which is the equation we were looking for. Since $m_{0}\left(x_{s}\right)$, $m_{1}\left(x_{s}\right)$, and $p_{1}\left(x_{s}\right)$ can be taken directly from Eqs. (51), (52), and (66), respectively, we can proceed to the final result. Indeed, using Eqs. (51), (52), (66) in (71), we obtain

$\frac{x_{s}}{m_{s}}=\frac{9}{4}-1.529 \alpha^{2}$,

up to order $\alpha^{2}$.

In converting from the variable $x$ back to $r$, we use

$\frac{x_{s}}{m\left(x_{s}\right)}=\frac{R}{M}$.

In addition, it can also be interesting to express $\alpha$ in terms of the total charge $Q$ and mass $M$. The following relation valid for small $q_{1}$, or small $\alpha$, can be found: $\frac{Q}{M}=\frac{q_{1}\left(x_{s}\right)}{m_{0}\left(x_{s}\right)+m_{1}\left(x_{s}\right)}=$ $\frac{q_{1}\left(x_{s}\right)}{m_{0}\left(x_{s}\right)}$, so that

$\frac{q_{1}\left(x_{s}\right)}{m_{0}\left(x_{s}\right)}=\frac{Q}{M}$

up to order $q_{1}$. We can then express $\alpha$ in terms of the ratio $Q / M$. Using Eqs. (50) and (51) in Eq. (74), and solving the resulting equation for $\alpha$, we obtain

$1.641 \alpha=\frac{Q}{M}$ 
4.2 The electric interior Schwarzschild limit: the $R, M$ and $Q$ relation for small charge

We are now in a position to calculate the desired ratio (72) in terms of the quantities $R, M$ and $Q$ and find the appropriate relation. Inserting Eq. (73) into Eq. (72) we find

$$
\frac{R}{M}=\frac{9}{4}-1.529 \alpha^{2},
$$

which is one form of the interior Schwarzschild limit for small charge.

Inserting Eq. (75) into Eq. (76) we get

$$
\frac{R}{M}=\frac{9}{4}-0.568 \frac{Q^{2}}{M^{2}},
$$

valid up to order $Q^{2} / M^{2}$. This is another form of the interior Schwarzschild limit for small charge.

We can also express the limit in terms of the horizon radius, $r_{+}$, for the Reissner-Nordström metric. The horizon radius is defined by Eq. (19), i.e., up to order $Q^{2} / M^{2}$ one has, $r_{+}=M+\sqrt{M^{2}-Q^{2}}=2 M\left(1-\frac{1}{4} \frac{Q^{2}}{M^{2}}\right)$. So,

$$
\frac{R}{r_{+}}=\frac{9}{8}-0.003 \frac{Q^{2}}{M^{2}}
$$

up to order $Q^{2} / M^{2}$. Equation (78) is yet another form of the interior Schwarzschild limit for small charge.

The electric interior Schwarzschild limit for small charge presented in various forms in Eqs. (76), (77), and (78) is the main result of this work. All the three forms of the electric interior Schwarzschild limit for small charge show that, in comparison with the uncharged case, Eq. (37), the star can be more compact. In particular, Eq. (78) shows that in the charged case the radius of the star can be a bit closer to its own gravitational radius.

In [44] these compact stars were studied numerically. An $R / M \times Q / M$ relation was given numerically for $0 \leq$ $Q / M \leq 1$. For small charge, $Q / M \ll 1$, one can extract from the numerical calculations in [44] that $\frac{R}{M} \simeq 2.25-$ $0.6 \frac{Q^{2}}{M^{2}}$. This should be compared to our analytical calculation valid in first order of $Q / M$, given here in Eq. (77), i.e., $\frac{R}{M}=\frac{9}{4}-0.568 \frac{Q^{2}}{M^{2}}$. It shows that the numerical code used in [44] is compatible with the analytical calculation. In that work [44] it was also shown numerically that in the other extreme, namely, $Q / M=1$, one would obtain a star at its own gravitational radius, $R / M=1$, i.e., an (extremal) quasiblack hole.

A related theme is the Buchdahl and the BuchdahlAndréasson bounds. Buchdahl [53], by imposing a simple set of assumptions, namely, that the spacetime is spherically symmetric, the star is made of a perfect fluid, and the density is a nonincreasing function of the radius, found that the radius to mass relation is $R / M \geq 9 / 4$. Thus the Schwarzschild limit [33], i.e., the limiting $R / M$ configuration when the central pressure goes to infinity, is an instance that saturates the Buchdahl bound. Following the line of reasoning of Buchdahl, Andréasson [58] obtained a bound for the minimum radius of a star using the energy condition $p+2 p_{T} \leq \rho$ where $p_{T}$ is the tangential pressure, $p$ is the radial pressure and $\rho$ is the energy density. This bound, the Buchdahl-Andréasson bound, is given by $\frac{R}{M} \leq \frac{9}{\left(1+\sqrt{1+3 Q^{2} / R^{2}}\right)^{2}}$. Retaining terms in first order in $Q^{2}$ one gets $\frac{R}{M}=\frac{9}{4}-0.667 \frac{Q^{2}}{M^{2}}$. Thus, our configurations of constant density and a charge distribution proportional to the energy density having $\frac{R}{M}=\frac{9}{4}-0.568 \frac{Q^{2}}{M^{2}}$, see Eq. (77), does not saturate the bound. This raises the question of whether there are other types of charged matter that can saturate the bound. One type is thin shells with an appropriate relation between the surface energy density and surface pressure [58]. Are there continuous (non-thin-shell) distribution configurations that saturate the bound? It seems that, as the configurations analyzed here, the configurations studied in [41-43] do not saturate the bound. It remains to be seen if the electrically charged configurations analyzed in $[48,49]$ saturate the bound. For further study of the bounds of electrically charged stars, see [59-61].

\subsection{A mass bound}

We can adapt the mass bound from Sect. 3.3 to the small charge case. Indeed, from Eq. (42) and the definition $M \equiv$ $m(R)$, we have at the boundary

$M=m_{0}(R)+m_{1}(R)$.

Now, Eq. (51) at the boundary can be put in the form $m_{0}(R)=$ $\frac{1}{2} R_{c} \sin ^{3} \chi_{s}$. So, Eq. (79) yields

$M=\frac{1}{2} R_{c} \sin ^{3} \chi_{s}+m_{1}(R)$.

Since $p_{c} \leq \infty$, using Eq. (68) with $p_{1}(R)=0$, one obtains $\sin ^{2} \chi_{s} \leq \frac{8}{9}$, and the bound for the non-perturbed mass $m_{0}$ is given by $m_{0}(R)=\frac{1}{2} R_{c} \sin ^{3} \chi_{s} \leq \frac{1}{2}\left(\frac{8}{9}\right)^{3 / 2} R_{c}$. In order to obtain the bound for $m_{1}$, we have to substitute the bound $\sin ^{2} \chi_{s} \leq \frac{8}{9}$ in Eq. (52). This is enough since we are only working up to order $\alpha^{2}$. Thus, the mass bound for the small charge case is

$$
M \leq \frac{1}{2}\left(\frac{8}{9}\right)^{3 / 2} R_{c}\left(1+0.679 \alpha^{2}\right)
$$


In the case of a compact star composed of neutrons in the incompressible state speckled with some charged particles, we obtain

$$
M \leq 1.5 M_{\odot}\left(1+0.679 \alpha^{2}\right)
$$

Comparing Eqs. (82) and (40) we see that we can attain a bigger mass on a charged star. This is expected since the electrostatic repulsion is opposite to the gravitational force, which means that we can put more mass on the star without it collapsing.

\section{Conclusions}

In this work we have studied compact stars with a small electric charge and found the limiting radius to mass relation and the maximum mass through an analytical approach based on Volkoff and Misner's method to solve the TOV equation for incompressible matter. More specifically, we have analyzed the interior Schwarzschild limit of spherically symmetric star configurations composed of a fluid with constant energy density $\rho$ and with a small electrical charge distribution $\rho_{e}$ proportional to $\rho, \rho_{e}=\alpha \rho$ with $\alpha \ll 1$. The exterior spacetime is described by the Reissner-Nordström metric. We have found through our analytical scheme that due to the electric charge distribution the limiting star configuration can have more mass and a smaller radius relatively to the limiting star with zero charge. This is expected since the electric charge distribution has a repulsive effect, adding to the pressure as a force that withstands the star. For stars containing some type of dark matter in their interiors there is the possibility that they possess a small but non-negligible electric charge, in which case our analytical formula is opportune and can be confronted quantitatively with observational data.

Acknowledgments We thank FCT-Portugal for financial support through Project No. PEst-OE/FIS/UI0099/2014. JPSL and VTZ thank CAPES-Brazil, for support within the Programa CSF-PVE, Project No. 88887.068694/2014-00.

Open Access This article is distributed under the terms of the Creative Commons Attribution License which permits any use, distribution, and reproduction in any medium, provided the original author(s) and the source are credited.

Funded by $\mathrm{SCOAP}^{3}$ / License Version CC BY 4.0.

\section{Appendix A: Behavior of the perturbed pressure for small radius}

Our goal is to compute the limit of infinite central pressure $p(r=0)$ in this charged case, so it is important to analyze the behavior of $p_{1}$ for small radius $x, x \sim 0$, which also means $\chi \sim 0$, and $r \sim 0$. We start by obtaining the perturbed charge, $q_{1}$, for a small radius expanding Eq. (50), which gives

$q_{1}(x) \sim \alpha \frac{x^{3}}{2}$

and the perturbed mass, $m_{1}$, from Eq. (52)

$m_{1}(x) \sim \alpha^{2} \frac{3}{20} x^{5}$

The non-perturbed pressure, $p_{0}$, given by Eq. (61) becomes

$p_{0}(x) \sim p_{0}(x=0)-\frac{\sqrt{1-x_{s}^{2}}}{3 \sqrt{1-x_{s}^{2}}-1} x^{2}$.

Using Eq. (56) for the perturbed pressure, $p_{1}$, together with Eqs. (A.1), (A.2), and (61) gives

$\frac{\mathrm{d} p_{1}}{\mathrm{~d} x} \sim-p_{1}\left(3 p_{0}(x=0)+2\right) x+\frac{1}{2} \alpha^{2} x$.

Integrating Eq. (A.4) one obtains

$$
\begin{aligned}
& p_{1}(x) \sim \frac{\alpha^{2}}{6 p_{0}(x=0)+4} \\
& +\left(p_{1}(x=0)-\frac{\alpha^{2}}{6 p_{0}(x=0)+4}\right) e^{-\left(3 p_{0}(x=0)+2\right) x^{2} / 2}
\end{aligned}
$$

So the unique way that the central pressure $p(r)=p_{0}(r)+$ $p_{1}(r)$ blows up is $p_{0}(r=0)$ blowing up.

\section{References}

1. S. Chandrasekhar, The maximum mass of ideal white dwarfs. Astrophys. J. 74, 81 (1931)

2. L.V. Landau, On the theory of stars. Physikalische Zeitschrift der Sowjetunion 1, 285 (1932)

3. J.R. Oppenheimer, G. Volkoff, On massive neutron cores. Phys. Rev. 55, 374 (1939)

4. B.K. Harrison, K.S. Thorne, M. Wakano, J.A. Wheeler, Gravitation Theory and Gravitational Collapse (University of Chicago Press, Chicago, 1965)

5. N.K. Glendenning, Compact Stars: Nuclear Physics, Particle Physics, and General Relativity, 2nd edn. (Springer, Berlin, 2000)

6. V. Rezania, R. Maartens, Vorticity affects the stability of neutron stars. Phys. Rev. Lett. 84, 2560 (2000). arXiv:gr-qc/9909073

7. M. Milgrom, A modification of the Newtonian dynamics as a possible alternative to the hidden mass hypothesis. Astrophys. J. 270, 365 (1983)

8. J. Bekenstein, M. Milgrom, Does the missing mass problem signal the breakdown of Newtonian gravity? Astrophys. J. 286, 7 (1984)

9. M. Milgrom, MOND theory. Can. J. Phys. 93, 107 (2015). arXiv:1404.7661 [astro-ph.CO]

10. B. Famaey, S.S. McGaugh, Modified Newtonian dynamics (MOND): observational phenomenology and relativistic extensions. Living Rev. Relativ. 15, 1 (2012). arXiv:1112.3960 [astroph.CO] 
11. C. Kouvaris, P. Tinyakov, Can neutron stars constrain dark matter? Phys. Rev. D 82, 063531 (2010). arXiv:1004.0586 [astro-ph.GA]

12. C. Kouvaris, M.A. Perez-Garcia, Can dark matter explain the braking index of neutron stars? Phys. Rev. D 89, 103539 (2014). arXiv:1401.3644 [astro-ph.SR]

13. P. Pani, E. Berti, V. Cardoso, J. Read, Compact stars in alternative theories of gravity. Einstein-Dilaton-Gauss-Bonnet gravity. Phys. Rev. D 84, 104035 (2011). arXiv:1109.0928 [gr-qc]

14. T. Harko, F.S. N. Lobo, M.K. Mak, S.V. Sushkov, Structure of neutron, quark, and exotic stars in Eddington-inspired Born-Infeld gravity. Phys. Rev. D 88, 044032 (2013). arXiv:1305.6770 [gr-qc]

15. C. Deliduman, K.Y. Ekşi, V. Keleş, Neutron star solutions in perturbative quadratic gravity. JCAP 05, 036 (2012). arXiv:1112.4154 [gr-qc]

16. S.S. Yazadjiev, D.D. Doneva, K.D. Kokkotas, K.V. Staykov, Nonperturbative and self-consistent models of neutron stars in Rsquared gravity. JCAP 06, 003 (2014). arXiv:1402.4469 [gr-qc]

17. M..K. Cheoun, C. Deliduman, C. Güngör, V. Keleş, C.Y. Ryu, T. Kajino, G.J. Mathews, Neutron stars in a perturbative $f(R)$ gravity model with strong magnetic fields. JCAP 10, 021 (2013). arXiv:1304.1871 [astro-ph.HE]

18. A.V. Astashenok, S. Capozziello, S.D. Odintsov, Further stable neutron star models from $\mathrm{f}(\mathrm{R})$ gravity. JCAP 12, 040 (2013). arXiv:1309.1978 [gr-qc]

19. N. Deruelle, Stars on branes: the view from the brane, in Proceedings in honor of Prof. Tomita (2011). arXiv:gr-qc/0111065

20. C. Germani, R. Maartens, Stars in the braneworld. Phys. Rev. D 64, 124010 (2001). arXiv:hep-th/0107011

21. J. Hladík, Z. Stuchlík, Photon and neutrino redshift in the field of braneworld compact stars. JCAP 07, 012 (2011). arXiv: 1108.5760 [gr-qc]

22. L.B. Castro, M.D. Alloy, D.P. Menezes, Mass radius relation of compact stars in the braneworld. JCAP (to appear) (2014). arXiv:1403.1099 [nucl-th]

23. D.J. Kaup, Klein-Gordon geon. Phys. Rev. 172, 1331 (1968)

24. R. Ruffini, S. Bonazzola, Systems of self-gravitating particles in general relativity and the concept of an equation of state. Phys. Rev. 187, 1767 (1969)

25. T.D. Lee, Soliton stars and the critical masses of black holes. Phys. Rev. D 35, 3637 (1987)

26. P. Jetzer, Boson stars. Phys. Rep. 220, 163 (1992)

27. S.L. Liebling, C. Palenzuela, Dynamical boson stars. Living Rev. Relati. 15, 6 (2012). arXiv:1202.5809 [gr-qc]

28. T. Tamaki, N. Sakai, Gauged Q-balls in the Affleck-Dine mechanism (2014). arXiv:1401.0996 [hep-th]

29. M.P. Dabrowski, F.E. Schunck, Boson stars as gravitational lenses. Astrophys. J. 535, 316 (1998). arXiv:astro-ph/9807039

30. Y.-F. Yuan, R. Narayan, M.J. Rees, Constraining alternate models of black holes: type I X-ray bursts on accreting fermionfermion and boson-fermion Stars. Astrophys. J. 606, 1112 (2004). arXiv:astro-ph/0401549

31. C. Palenzuela, L. Lehner, S.L. Liebling, Orbital dynamics of binary boson star systems. Phys. Rev. D 77, 044036 (2008). arXiv:0706.2435 [gr-qc]

32. S. Hannestad, A. Ringwald, H. Tu, Y.Y.Y. Wong, Is it possible to tell the difference between fermionic and bosonic hot dark matter? JCAP 05, 09 (2014). arXiv:astro-ph/0507544

33. K. Schwarzschild, Uber das Gravitationsfeld eines Kugel aus inkompressibler Flüssigkeit nach der Einsteinschen Theorie. Sitzungsberichte der Königlich Preussischen akademie der Wissenschaften 1, 424 (1916); K. Schwarzschild (translation by Antoci, S.), On the gravitational field of a sphere of incompressible fluid according to Einstein's theory. arXiv:physics/9912033

34. G.M. Volkoff, On the equilibrium of massive spheres. Phys. Rev. $\mathbf{5 5}, 413$ (1939)
35. C.W. Misner, Gravitational collapse, in Astrophysics and General Relativity, Brandeis University Summer School in Theoretical Physics, ed. by M. Chrétien, S. Deser, J. Goldstein (Gordon and Breach, New York, 1968), p. 113

36. R.C. Tolman, Static solutions of Einstein's field equation for spheres of fluid. Phys. Rev. D 55, 364 (1939)

37. M.K. Mak, T. Harko, Isotropic stars in general relativity. Eur. Phys. J. C 73, 2585 (2013). arXiv:1309.5123 [gr-qc]

38. L. Iorio, Constraining the electric charges of some astronomical bodies in Reissner-Nordström spacetimes and generic $r^{-2}$-type power-law potentials from orbital motions. Gen. Relativ. Gravit. 44, 1753 (2012)

39. M. Byrne, C. Kolda, P. Regan, Bounds on charged, stable superpartners from cosmic ray production. Phys. Rev. D 66, 075007 (2002). arXiv:hep-ph/0202252

40. J. Bekenstein, Hydrostatic equilibrium and gravitational collapse of relativistic charged fluid balls. Phys. Rev. D 4, 2185 (1971)

41. F. de Felice, Y.-Q. Yu, J. Fang, Relativistic charged spheres. Mon. Not. R. Astron. Soc. 277, L17 (1995)

42. F. de Felice, S.-M. Liu, Y.-Q. Yu, Relativistic charged spheres: II. Regularity and stability. Class. Q. Grav. 16, 2669 (1999). arXiv:gr-qc/9905099

43. P. Anninos, T. Rothman, Instability of extremal relativistic charged spheres. Phys. Rev. D 65, 024003 (2002). arXiv:gr-qc/0108082

44. J.D.V. Arbañil, J.P.S. Lemos, V.T. Zanchin, Incompressible relativistic spheres: electrically charged stars, compactness bounds, and quasiblack hole configurations. Phys. Rev. D 89, 104054 (2014). arXiv:1404.7177 [gr-qc]

45. S. Ray, A.L. Espíndola, M. Malheiro, J.P.S. Lemos, V.T. Zanchin, Electrically charged compact stars and formation of charged black holes. Phys. Rev. D 68, 084004 (2003). arXiv:astro-ph/0307262

46. C. Ghezzi, Relativistic structure, stability, and gravitational collapse of charged neutron stars. Phys. Rev. D 72, 104017 (2005). arXiv:gr-qc/0510106

47. J.D.V. Arbañil, J.P.S. Lemos, V.T. Zanchin, Polytropic spheres with electric charge: compact stars, the Oppenheimer-Volkoff and Buchdahl limits, and quasiblack holes. Phys. Rev. D 88, 084023 (2013). arXiv:1309.4470 [gr-qc]

48. B.S. Guilfoyle, Interior Weyl-type solutions to the EinsteinMaxwell field equations. Gen. Relativ. Gravit. 31, 1645 (1999). arXiv:gr-qc/9906089

49. J.P.S. Lemos, V.T. Zanchin, Quasiblack holes with pressure: relativistic charged spheres as the frozen stars. Phys. Rev. D 81, 124016 (2010). arXiv: 1004.3574 [gr-qc]

50. J.P.S. Lemos, E. Weinberg, Quasiblack holes from extremal charged dust. Phys. Rev. D 69, 104004 (2004). arXiv:gr-qc/0311051

51. P. Jetzer, P. Liljenberg, B.-S. Skagerstam, Charged boson stars and vacuum instabilities. Astropart. Phys. 1, 429 (1993). arXiv:astro-ph/930501

52. Y. Brihaye, V. Diemer, B. Hartmann, Charged Q-balls and boson stars and dynamics of charged test particles. Phys. Rev. D 89, 084048 (2014). arXiv:1402.1055 [gr-qc]

53. H.A. Buchdahl, General relativistic fluid spheres. Phys. Rev. 116, 1027 (1959)

54. H. Bondi, Massive spheres in general relativity. Mon. Not. R. Astron. Soc. 282, 303 (1964)

55. J.N. Islam, Some general relativistic inequalities for a star in in hydrostatic equilibrium. Mon. Not. R. Astron. Soc. 145, 21 (1969)

56. J.N. Islam, Some general relativistic inequalities for a star in in hydrostatic equilibrium II. Mon. Not. R. Astron. Soc. 147, 377 (1970)

57. D. Martin. M. Visser, Bounds on the interior geometry and pressure profile of static fluid. Class. Q. Gravit. 20, 3699 (2003). arXiv:gr-qc/0306038 
58. H. Andréasson, Sharp bounds on the critical stability radius for relativistic charged spheres. Commun. Math. Phys. 288, 715 (2009). arXiv:0804.1882 [gr-qc]

59. Y.-Q. Yu, S.-M. Liu, Relativistic charged balls. Commun. Theor. Phys. 33, 571 (2000). arXiv:gr-qc/9904050

60. C. G. Böhmer, T. Harko, Minimum mass-radius ratio for charged gravitational objects. Gen. Relativ. Gravit. 39, 757 (2007). arXiv:gr-qc/0702078

61. A. Giuliani, T. Rothman, Absolute stability limit for relativistic charged spheres. Gen. Relativ. Gravit. 40, 1427 (2008). arXiv:0705.4452 [gr-qc]
62. W. Israel, Dark stars: the evolution of an idea, in Three Hundred Years of Gravitation, ed. by S.W. Hawking, W. Israel (Cambridge University Press, Cambridge, 1987), p. 199

63. W.B. Bonnor, Equilibrium of charged dust in general relativity. Gen. Relativ. Gravit. 12, 453 (1980)

64. J.P.S. Lemos, V.T. Zanchin, Bonnor stars in d spacetime dimensions. Phys. Rev. D 77, 064003 (2008). arXiv:0802.0530 [gr-qc] 